Supporting Information

\title{
Hydrothermal decomposition of glucose in the presence of ammonium
}

Ivan Kristianto, Brian S. Haynes, and Alejandro Montoya*

School of Chemical and Biomolecular Engineering, The University of Sydney, Sydney, New South Wales 2006, Australia 




Figure S1. Typical GC-MS chromatogram of DCM-extracted fraction from hydrothermal reaction of GLU/AmA. Reaction condition: $280{ }^{\circ} \mathrm{C}$, 1s, and initial GLU concentration was $55.5 \mathrm{mmol} / \mathrm{L}$ with $\lambda_{G L U / A m A}=1 / 1.6$. 




Figure S2. Typical HPLC/UV response of GLU/AmA decomposition at $260 \mathrm{~nm}$ using C-18 column. Reaction condition: $260^{\circ} \mathrm{C}, 1 \mathrm{~s}$, and GLU/AmA $=1 / 1.6$ with the initial concentration of GLU was $55.5 \mathrm{mmol} / \mathrm{L}$. 
Table S1. Absorbance measurement at $420 \mathrm{~nm}$ of product mixture at various a) $\lambda_{G L U / A m A}$ at $\left.280{ }^{\circ} \mathrm{C}, 1 \mathrm{~s}, \mathrm{~b}\right)$ residence time, and (c) reaction temperature

a)

\begin{tabular}{ccc}
\hline Initial glucose concentration $(\mathrm{mmol} / \mathrm{L})$ & $\lambda_{\text {GLU } / A m A}$ & $\mathrm{Abs}_{420 \mathrm{~nm}}$ \\
\hline & $1 / 0$ & 0 \\
\multirow{2}{*}{5.6} & $1 / 1.6$ & 0.004 \\
& $1 / 6.4$ & 0.006 \\
& $1 / 12$ & 0.008 \\
& $1 / 15$ & 0.012 \\
\hline \multirow{2}{*}{55.5} & $1 / 0.3$ & 0.038 \\
& $1 / 1.1$ & 0.099 \\
& $1 / 1.6$ & 0.159 \\
\hline
\end{tabular}

b)

\begin{tabular}{cc}
\hline Residence Time (s) & Abs $_{420 \mathrm{~nm}}$ \\
\hline 0.5 & 0.06 \\
1 & 0.106 \\
1.5 & 0.132 \\
2 & 0.176 \\
\hline
\end{tabular}

c)

\begin{tabular}{cc}
\hline Reaction Temperature $\left({ }^{\circ} \mathrm{C}\right)$ & $\mathrm{Abs}_{420 \mathrm{~nm}}$ \\
\hline 220 & 0.011 \\
240 & 0.042 \\
260 & 0.106 \\
280 & 0.159 \\
\hline
\end{tabular}




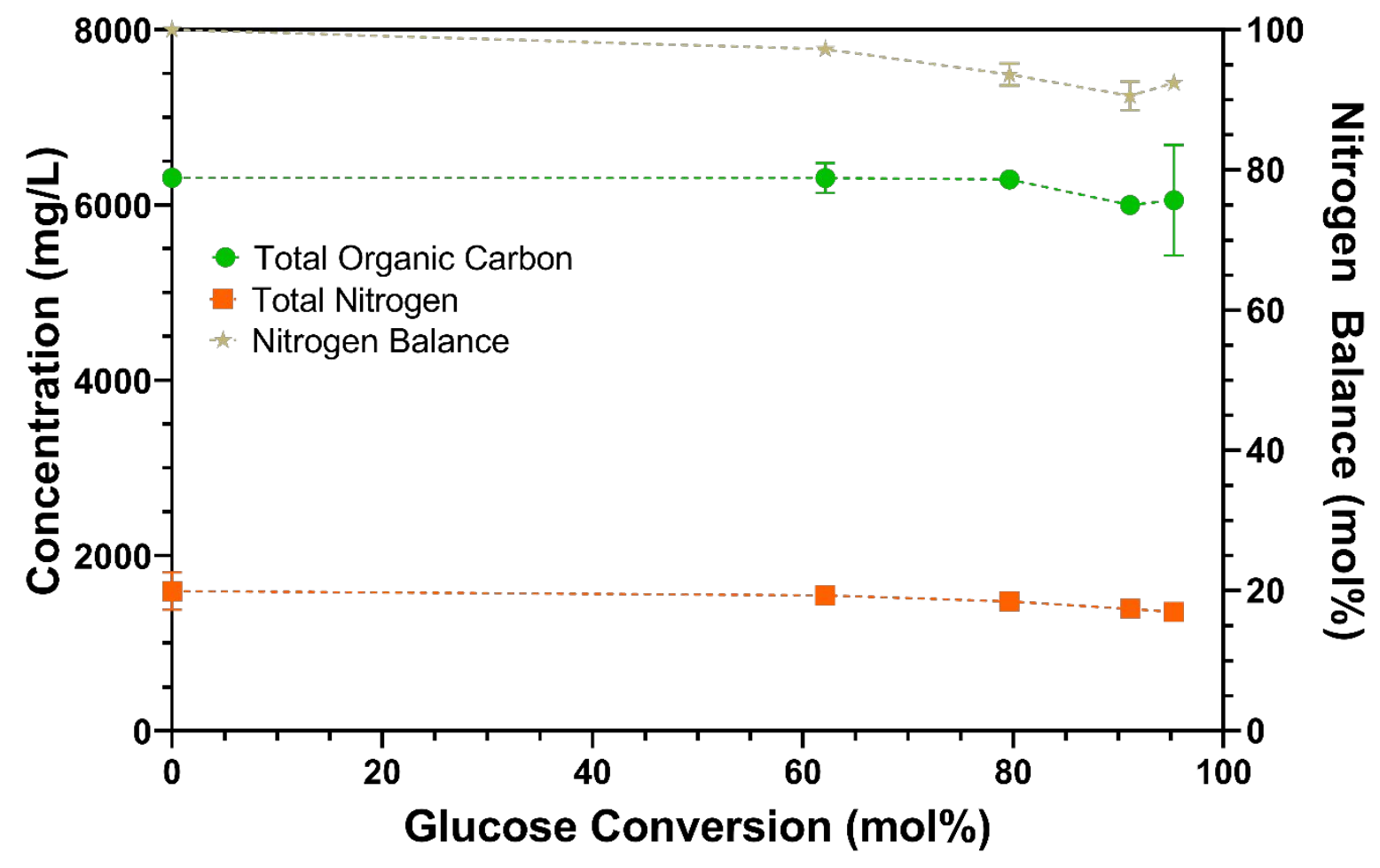

Figure S3. Total Organic Carbon (TOC), Total Nitrogen (TN), and Nitrogen Balance as a function GLU conversion at varying residence times at $260{ }^{\circ} \mathrm{C}$. $\lambda_{G L U / A m A}$ is fixed at $1 / 1.6$ with the initial glucose concentration of $55.5 \mathrm{mmol} / \mathrm{L}$. 


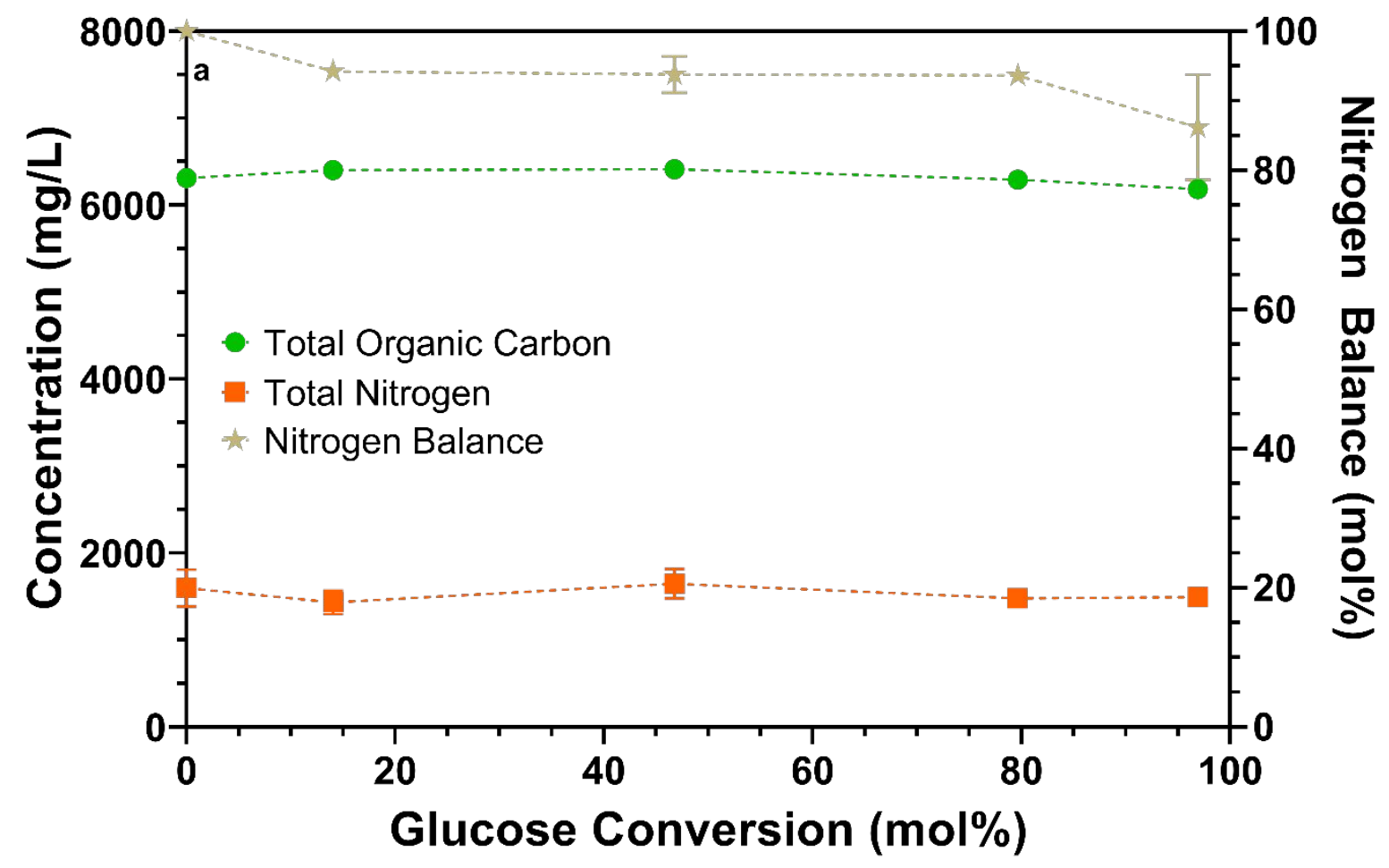

Figure S4. Total Organic Carbon (TOC), Total Nitrogen (TN), and Nitrogen Balance as a function GLU conversion at varying reaction temperature at $260{ }^{\circ} \mathrm{C}$. $\lambda_{G L U / A m A}$ is fixed at 1/1.6 with the initial glucose concentration of $55.5 \mathrm{mmol} / \mathrm{L}$ 


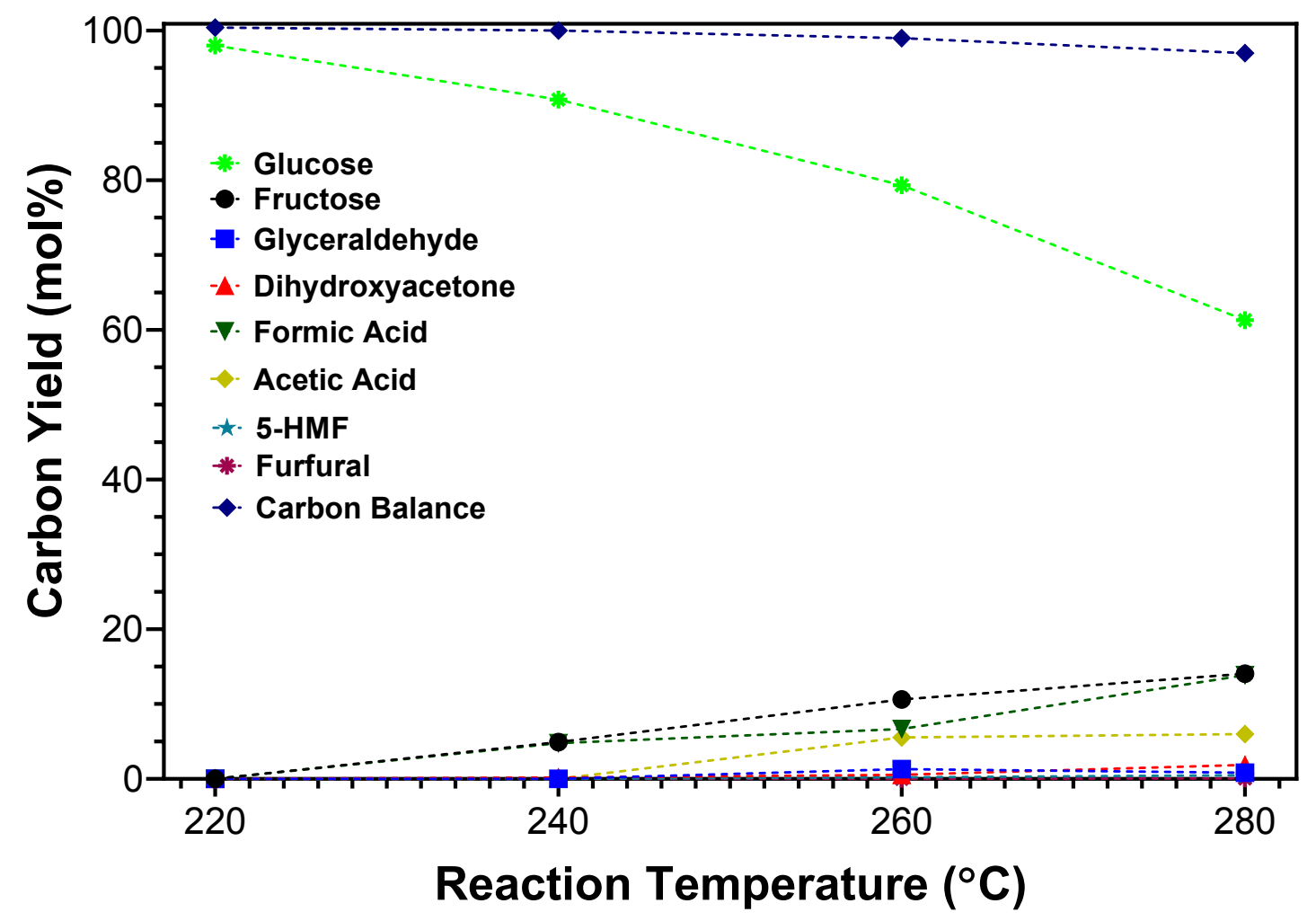

Figure S5. Carbon yields of GLU, FRU, GCA, DHA, FA, AA, 5-HMF, and FF as a function of reaction temperature, produced from GLU decomposition at $1 \mathrm{~s}$ in the absence of AmA with initial GLU concentration: $55.5 \mathrm{mmol} / \mathrm{L}$. 